\title{
Socially Engaged Social Psychology in Asia: Sustaining Research Progress in Diverse Directions
}

\author{
Allan B. I. Bernardo ${ }^{1}$ and James H. Liu² \\ 1 University of Macau, Macau \\ ${ }^{2}$ Massey University, Auckland, New Zealand
}

\begin{abstract}
The final thematic special issue in the series 'The Social Psychology of Social Change: Science and Practice in Asia' features 10 empirical studies that apply diverse social psychological theories and approaches to understand and to address a wide range of social concerns in Asian societies. The articles in the special issue align more to mainstream social psychological approaches to studying social phenomena, and as such are mainly derivative of Western social psychological paradigms, with the exception of a couple of studies that adopt emic indigenous approaches. Nevertheless, the various studies display a clear motivation to use social psychology to engage particular societal concerns, representing incremental progress towards using social psychology in the service of social change.
\end{abstract}

Keywords: applied social psychology, social change, social concerns, Asian societies

This thematic Special Issue on applied social psychology research in Asia is the last in the series entitled 'The Social Psychology of Social Change: Science and Practice in Asia'. The series was intended to provide a platform for social psychological research aiming to solve social problems while generating new knowledge; as such, the initial call for papers emphasised the theoretical and practical approach that had culture and social change at its core (Liu \& Bernardo, 2014). This aspiration was later more fully articulated in Liu's proposal for Height psychology (Liu, 2017), which is most consistent with the approach of social psychologists more engaged in indigenous psychological approaches (Bhawuk, 2017). The first special issue in this series featured some action research projects that were drawn from indigenous social concepts (Liu et al., 2014; $\mathrm{Su}, \mathrm{Pan}, \&$ Chen, 2014), and as such, the series seemed to show promise in nurturing this social change orientation in social psychology research in Asia. But in the following years, it became increasingly difficult to gather action research studies or studies that were oriented towards social change. Instead, the articles in the special issues tended to focus more on describing and theorising about specific social phenomena and social concerns in particular Asian societies (Liu \& Bernardo, 2016).

Nevertheless, these articles in the special issue series all represent some progress towards engaging social psychology research with societal concerns, particularly concerns that seem to arise from different forces of social change. Thus, even as the series was not able to encourage more action-oriented research on the psychology of social change and cultural change, there were clear positive steps towards using social psychological theories and methods to better understand social concerns and to develop psychological programs that address such concerns. Even as there have been rather limited works that aimed to appropriate and elaborate indigenous social philosophies, concepts, and methods, there has been a clear attempt to ground social psychology research in social experiences of Asian peoples. This is most evident in research that directly inquires into social problems and that takes an applied social psychological approach (Bernardo \& Liu, 2015). This focus and approach is continued in this special issue, as we see research on very timely social concerns, which includes inclusiveness and access for disabled persons (Miyahara et al., this issue), socioeconomic inequality (Reyes, this issue), stress related to internal migration (English \& Worlton, 2017), corruption among government officials (Xu et al., this issue), intentions to rebel (Chen et al., this issue), and drug prevention (Hechanova et al., this issue), among others.

The attempt to ground social psychology in Asian social experiences is also exemplified in the approach of using cultural lenses in describing and theorising about these social concerns (Bernardo \& Liu, 2015). Most of the studies in the special issue anchor the inquiry on established (Western) social psychological theories and concepts, but 
extend and modify these theories by adding a cultural dimension either by studying how cultural factors moderate the theoretical relationships (see, e.g., Reyes, this issue) or by drawing from cultural explanations to make sense of unexpected findings (see, e.g., Lin, Huang, Chen, \& Pakpour, this issue). Two articles in the special issue exemplify this cultural approach by applying an emic approach to studying corruption (Xu et al., this issue) and vocational experiences (Kang, Wang, Xi, \& Pang, this issue) in Chinese societies.

These efforts to undertake applied social psychology research, although still more basic descriptive and theoretical research, nevertheless represent an attempt to be more socially engaged. The value in these attempts can be best appreciated by reading the 10 contributions in this special issue, which we describe in the following sections.

The first article in this special issue was actually published earlier in 2017. English and Worlton's (2017) article is a longitudinal study on the uprooting stress experienced by educational Chinese migrants. As Chinese society continues to grow its knowledge-driven economy, higher education has become increasingly important to Chinese people, and more students choose to migrate to the bigger cities or other countries where there are better opportunities to pursue higher learning. The study inquired into the uprooting stress experiences, primary and secondary coping, and anxiety at two time points of educational migrants within China. One of the most interesting aspects of the results was related to how primary coping interacted with uprooting stress experiences. In particular, primary coping strategies predicted higher anxiety levels later in the semester among those who experienced high uprooting stress. Their results also revealed strong gender differences in anxiety. English and Worlton refer to the goodness-of-fit hypothesis in explaining why primary but not secondary coping relates to uprooting stress and anxiety, but are mindful of the fact that they did not consider cultural differences associated with educational migration from rural versus urban residences. The application of cultural dimensions of the goodness-of-fit hypotheses in understanding how educational migrants adjust to their uprooting experiences has important implications for discussions about the wellbeing of this growing section of the Chinese population, including those who migrate to other countries for their higher education (Cao, Zhu, \& Meng, 2017).

The second article in the special issue also has a mental health and wellbeing focus. Lin et al. (this issue) report the results of a retrospective longitudinal study involving a sample of patients with schizophrenia. The study inquired into the relationship between self-stigma and subjective quality of life of the patients, and focused on the mediating role of self-esteem in this relationship. Although the results of the study indicated the negative impact of self-stigma on the patients' self-esteem, the mediating role of self-esteem on subjective quality of life was only partially supported by testing the mediation model. In their discussion of this result, which diverges slightly from the extant literature, Lin and colleagues refer to (among other factors) the debates on the importance of self-esteem in East Asian cultures (Heine, Lehman, Markus, \& Kitayama, 1999). Their conclusion affirms the vital role of self-esteem in psychological outcomes even among East Asian cultures, but that self-stigma arising from the experience of patients with schizophrenia might exert an extremely strong influence on their quality of life. The role of self-esteem will continue to be the focus of debates on the cultural importance of the construct, as some studies point to its importance (e.g., Zhang, Luo, Zhao, Zhang, \& Wang, 2017), while other studies note the declining levels of self-esteem in different East Asian populations (e.g., Hamamura, 2017). Nevertheless, the authors suggest that mental health professionals should develop self-stigma reduction and self-esteem enhancement interventions to help their schizophrenic patients, underscoring the importance of social cognitive processes in addressing aspects of the wellbeing of patients.

Cultural difference was also one of the points of inquiry in the article in this special issue that related to prosocial or helping intentions towards people with disabilities. The specific measures of prosocial behavioural intentions in Miyahara et al.'s (this issue) study relate to emphatic concerns for disability and accessibility, but psychological principles involved could probably refer prosocial intentions to any social issue that relates to inclusivity in a diverse society. The main interest in the study was how gender and cultural differences (e.g., individualism vs. collectivism) might moderate emphatic concern by influencing the sense of interdependence in New Zealand and Japanese individuals. The first study showed how both gender and culture influenced emphatic concern but not interdependence. The mediation hypothesis was further pursued in the second study by priming selfconstruals. There was only partial support for the effectiveness of priming (i.e., only for priming independence in the Japanese sample) and partial indication for some relationship between interdependence and empathic concern (only among the Japanese participants). But the effects of gender and cultural difference on emphatic concern were still very evident. Thus, the study establishes how two factors influence empathic concern for disability and accessibility, although the proposed psychological mechanism was not supported by the data, and the authors point to the need to focus on other cultural sources that can account for these differences.

A different cultural dimension was the focus of the fourth article in the special issue. Reyes' study (this issue) looked into how cultural-level differences in power distance moderated attributions related to social inequality. Using East Asian and Pacific data of the International Social Survey Programme, the study was scaffolded on two previous findings: (a) that perceived social inequality leads to structural attributions of inequality, and (b) that higher subjective social position leads to more individualist attributions of inequality compared to lower subjective 
social position. By analysing cross-level interactions, Reyes demonstrated that the relationship between perceived social inequality and structural attributions was stronger in countries that were lower in power distance. On the other hand, the positive relationship between subjective social position and individualist attributions was stronger in countries that had higher power distance, among others. Reyes argues that the effects associated with contrasting power distance cultural environments influence the context within which individuals who have different perceptions of social inequality cognitively make sense of their relative positions in the unequal social space.

The fifth article in the special issue also deals with socioeconomic differences but does not make any explicit reference to cultural factors. Instead, Qi and Cui (this issue) demonstrate that the harmful effects of the thinideal on women's body image are actually associated with perceived socioeconomic status of representations of the thin-ideal. In their first study, Qi and Cui showed the negative relationships between exposure to thin-ideal images with Chinese womens' body and weight appreciation, and self-objectification. In their second study, they showed that images of thin-ideal that were not accompanied by luxurious accessories did not produce the same negative effects in the women's body image. Although the authors do not make very specific claims about the psychological mechanisms, they do suggest that the comparison processes that may underlie body self-image perceptions might go beyond mere shape comparisons and might actually be more strongly influenced by other forms of social comparisons. Social comparisons related to socioeconomic status might be particularly important in Chinese societies, which are currently undergoing rapid and sustained economic growth, and could be seen as a key aspect of the current cultural experiences in China. The findings of Qi and Cui seem to echo other research that shows the negative relationship between these materialist orientations and wellbeing in Asian societies (e.g., Nagpaul \& Pang, 2017).

The next two articles are perhaps the least 'cultural' among all the articles in the special issue, as the two studies do not explicitly refer to cultural variables or approach the social phenomena being studied from cultural lens. The sixth article anchors its explorations on the social construct of relative deprivation as a predictor of intentions to rebel among Chinese persons. Chen and colleagues (this issue) propose and test a model wherein individual relative deprivation directly relates to intentions to rebel, but also gives rise to a chain of other psychological processes (e.g., negative future expectation, lower social trust) that also relate to intentions to rebel. The proposed model and the results that support the model provide interesting theoretical applications of the relative deprivation concept; and it is of particular interest, given how individual relative deprivation is demonstrated to decrease collective forms of behaviour in a society that is known to be strongly collectivist. As Chen et al. suggest, the more important consequences of individual relative deprivation may relate to the mediating variables they studied. As has already been observed in some collectivist societies (Hamamura, 2017), decline in collective values such as social trust and future expectation might have other negative individual and social outcomes in the long term (see, e.g., Chen, 2017).

The seventh article is about how Chinese students accept career advice. The experimental studies by Kang et al. (this issue) explored the influence of regulatory orientation (prevention or promotion focus) on how career decision makers give more weight to advice that is presented using either an eager strategy or a vigilant strategy. They found no main effect either of regulatory focus or advice strategy, but they found an interaction effect that is consistent with a regulatory-fit effect. That is, participants in a promotion-focused condition gave more weight to the advice presented using the eager strategy, but those in the prevention-focused condition gave more weight to advice presented using the vigilant strategy. In their second experiment, they manipulated the participants' attribution (or non-attribution) of their feelings associated with regulatory fit. The results show that the earlier regulatory-fit effect disappears in the attribution condition. The results provide some nuanced elaborations on the psychological mechanisms underlying the regulatory-fit effect as applied to acceptance of career advice. Although there were no strongly cultural theoretical arguments in the study, Kang and co-authors underscore the significance of psychological processes involved in career and vocational decision making in Chinese society.

The eighth article in the special issue approaches the topic of vocation decision making from a more indigenous cultural perspective. Starting with a critical analysis of existing Western models of vocational interests and experiences, $\mathrm{Li}, \mathrm{Xu}, \mathrm{Li}$, and Zhang (this issue) construct a model of vocational experiences that derive from the experience of Mainland Chinese workers. The article describes the processes of developing the initial pool of items to express the range of vocational experiences of Chinese people, to identifying the dimensions of these vocational experiences using exploratory factor analytic procedures, to the confirmatory and validation processes that provide support for the meaningfulness of the identified dimensions of Chinese people's vocational experiences, and evidence for the good psychometric properties of the scale developed to measure these vocational experiences. Although using the standard quantitative methodologies in developing and validating psychological scales, the study of Li and co-authors is a clearly explicit attempt to develop theoretical models that are grounded on specific cultural experiences.

The ninth article in the special issue is another attempt to develop an indigenous model of a social phenomenon in China. Using a range of qualitative analyses of texts of corruption cases, $\mathrm{Xu}$ and colleagues (this issue) develop a model of the psychological mechanisms that underlie 
corruption (i.e., bribery) of Chinese government officials. From the results of their analyses, they propose a 'psychological kidnapping' model where the actors (bribers and government officials) in corruption first establish guanxi, then proceed through different stages (attraction and acceptance, trust and integration, and collusion or fracture) that are also characterised by distinct psychological processes (concealed resource delivery, imbalanced perceptions of corruption risk and cost for government officials, and soft menace from bribers). Xu and colleagues also identify renqing as another important indigenous concept that enables the psychological kidnapping model. Similar to the article by Li et al. (this issue), the attempt to develop an indigenous model in Xu et al.'s study was undertaken to provide newer theoretical bases to tackle a very critical social issue in current Chinese society that are hopefully more appropriate and relevant compared to the theoretical approaches that apply to Western models (e.g., Bai, Liu, \& Kou, 2016).

The final article in the special issue is also an attempt towards more culturally appropriate psychological interventions. Hechanova et al. (this issue) report on the development of a drug intervention program for low to mild drug users in the Philippines. The approach primarily drew from evidence-based approaches to drug recovery interventions, but the community-based aspects allowed for the research to draw from cultural and social psychological concepts and from indigenous social concepts to modify and adapt aspects of the intervention to be more suited to the Filipino community context. The article describes the process of developing the program, and the process itself reflects an attempt to draw from varied cultural stakeholder perspectives in designing and getting initial feedback on the intervention program. As the intervention program is still ongoing, the article does not go so far as to describe and evaluate the processes and outcomes, but it provides useful insights into how social psychological concepts and approaches can be used to guide more community mental health programs.

It is worth noting that this final special issue has the most number of articles and features the most diverse range of topics, theories, and methodological approaches among all the special issues in the 'The Social Psychology of Social Change' series. We would like to think that the increasing number reflects the momentum building towards more sustained applied social psychological research programs addressing a diverse range of societal concerns in Asia. The momentum is building but still in ways that align with and are derivative of mainstream Western social psychological sciences; inquiry is still anchored on social psychological theories and concepts developed by Western psychologists and mainly utilises positivist and post-positivist methodological approaches. Attempts to draw from indigenous philosophical systems or ways of knowing are still the minority. These trends may reflect how Asian cultural values, such as power distance and harmony in collectivism, may dissuade moves to chal- lenge dominant epistemologies in social psychology, and also how Asian academic institutions are increasingly embracing the norms and standards of excellence (see Liu \& Bernardo, 2016, for discussion).

But the range of articles in this special issue nevertheless displays a clear motivation to engage with social concerns and to develop theoretical frames for addressing these concerns, and the articles exemplify a diversity of approaches. There are studies that adopt the standard paradigms of mainstream experimental and quantitative social psychology to test hypotheses using Asian samples, and in doing so contribute to incremental elaborations or modifications of existing theories (see, e.g., Chen et al., this issue; English \& Worlton, 2017; Kang et al., this issue; Lin et al., this issue). There are studies that consider cultural and societal factors in contextualising the theoretical assumptions, then empirically test these culturally modified theoretical models (see e.g., Miyahara et al., this issue; Qi et al., this issue; Reyes, this issue) or rework psychological interventions in ways that take into account such cultural factors (e.g., Hechanova et al., this issue). And there are studies that take the emic approach and draw from indigenous concepts and experiences to develop models and theories to explain social psychological experiences of people in particular societies (see, e.g., Li et al., this issue; $\mathrm{Xu}$ et al., this issue). These diverse ways of being socially engaged are likely to co-exist in the work of Asian social psychologists for many more years, and should find support and make progress in the various social infrastructure of social psychology research. But perhaps there will be a time when the community of Asian social psychologists will decide that the progress has not been of the kind that allows social psychology to bring the true forms of social change needed in our communities.

\section{References}

Bai, B.Y., Liu, X.X., \& Kou, Y. (2016). Belief in a just world lowers bribery intention. Asian Journal of Social Psychology, 19, 66-75.

Bernardo, A.B., \& Liu, J.H. (2015). Sharpening our understanding of social problems in Asian societies: The roles of culture and theory in socially engaged social psychology. Journal of Pacific Rim Psychology, 9, 43-49.

Bhawuk, D.P. (2017). Developing theories and models to serve: A manifesto for indigenous psychologists. Asian Journal of Social Psychology, 20, 155-160.

Cao, C., Zhu, C., \& Meng, Q. (2017). Predicting Chinese international students' acculturation strategies from sociodemographic variables and social ties. Asian Journal of Social Psychology, 20, 85-96.

Chen, L. (2017). Applying the extended theory of planned behaviour to predict Chinese people's non-remunerated blood donation intention and behaviour: The roles of perceived risk and trust in blood collection agencies. Asian Journal of Social Psychology, 20, 221-231. 
Chen, X., Wang, X., Liu, J., Dong, S., Zhu, J., \& Huo, J. ( this issue). The relationship between individual relative deprivation and intention to rebel: A multiple mediation model. Journal of Pacific Rim Psychology, 12, e12. doi.10.1017/prp.2017.25.

English, A.S., \& Worlton, D.S. (2017). Coping with uprooting stress during domestic educational migration. Journal of $\mathrm{Pa}$ cific Rim Psychology, 11, e9. doi:10.1017/prp.2017.10

Hamamura, T. (2017). A cultural psychological analysis of cultural change. Asian Journal of Social Psychology. Advance online publication. doi:10.1111/ajsp.12194.

Hechanova, R., Alianan, A., Calleja, M., Melgar, I., Acosta, A., Villasanta, A., ... Cue, M. ( this issue). The development of a community-based drug intervention for Filipino drug users. Journal of Pacific Rim Psychology, 12, e12. doi.10.1017/prp.2017.23.

Heine, S.J., Lehman, D.R., Markus, H.R., \& Kitayama, S. (1999). Is there a universal need for positive self-regard? Psychological Review, 106, 766-794.

Kang, T., Wang, L., Xi, P., \& Pang, Q. ( this issue). Taking advise for vocational decisions: Regulatory fit effects. Journal of Pacific Rim Psychology, 12, e12. doi.10.1017/prp.2017.12.

Li, J., Xu, C., Li, C., \& Zhang, H. ( this issue). Construct and initial validation of a nine dimension vocational interests scale for young Chinese. Journal of Pacific Rim Psychology, 12, e12. doi.10.1017/prp.2017.26.

Lin, C., Huang, W., Chen, S., \& Pakpour, A. ( this issue). The mediation role of self-esteem for self-stigma on quality of life for people with schizophrenia. A retrospective longitudinal study. Journal of Pacific Rim Psychology, 12, e12. doi.10.1017/prp.2017.18.

Liu, J.H. (2017). Neo-Confucian epistemology and Chinese philosophy: Practical postulates for actioning psychology as a human science. Asian Journal of Social Psychology, 20, 137149.

Liu, J.H., \& Bernardo, A.B.I. (2014). Social psychology for social change: Foundations for and introduction to a program of action-oriented research. Journal of Pacific Rim Psychology, 8, 29-34.

Liu, J.H., \& Bernardo, A.B.I. (2016). What should a social psychology for social change look like in Asia? Reflections on scientific research aimed at solving social problems and generating new knowledge with a theory of culture change at its centre'. Journal of Pacific Rim Psychology, 10, e14. doi:10.1017/prp.2016.11

Liu, S.H., Wang, C.H., Deng, C.P., Keh, F.B., Lu, Y.J., \& Tsai, Y.C. (2014). Action research using a Chinese career model based on the wisdom of classic of changes and its applications. Journal of Pacific Rim Psychology, 8, 83-94.

Miyahara, M., Sawae, Y., Wilson, R., Briggs, H., Ishida, J., Dolhata, K., \& Sugiyama, A. ( this issue). An interdependence approach to empathic concern for disability and accessibility: Effects of gender, culture, and priming self-construal in Japan and New Zealand. Journal of Pacific Rim Psychology, 12, e12. doi.10.1017/prp.2017.19.

Nagpaul, T., \& Pang, J.S. (2017). Materialism lowers well-being: The mediating role of the need for autonomy-correlational and experimental evidence. Asian Journal of Social Psychology, 20, 11-21.

Qi, W., \& Cui, L. ( this issue). Being successful and being thin: The effects of thin-ideal social media images with high socioeconomic status on women's body image and eating behaviour. Journal of Pacific Rim Psychology, 12, e12. doi.10.1017/prp.2017.16.

Reyes, M.L. (this issue). Social mobility attributions in East Asian and Pacific cultures: Power distance and individualism as moderators of self-attribution bias. Journal of Pacific Rim Psychology, 12, e12. doi.10.1017/prp.2017.17.

Su, Y., Pan, R.J., \& Chen, K.H. (2014). Encountering selves and others: Finding meaning in life through action and reflection on a social service learning program. Journal of Pacific Rim Psychology, 8, 43-52.

Xu, Y., Jiao, L., Xu, R., Feng, Q., Wang, F., Jiang, J., \& Chen, C. (this issue). The process and characteristics of psychological kidnapping: An indigenous model of corruption in China. Journal of Pacific Rim Psychology, 12, e12. doi.10.1017/prp.2017.24.

Zhang, H., Luo, Y., Zhao, Y., Zhang, R., \& Wang, Z. (2017). Differential relations of grandiose narcissim and vulnerable narcissism to emotional dysregulation: Self-esteem matters. Asian Journal of Social Psychology, 20, 232237. 